\title{
Lithotomy versus prone position for perianal surgery: a randomized controlled trial
}

\author{
Pankaj Kumar, Tushar S. Mishra, Siddhant Sarthak, Prakash Kumar Sasmal \\ Department of General Surgery, All India Institute of Medical Sciences (AIIMS) Bhubaneswar, Bhubaneswar, India
}

Purpose: Studies objectively comparing lithotomy and prone positions regarding surgeon comfort, ergonomics, patient comfort, and position related complications are scarce.

Methods: The patients posted for surgery of either fistula in ano, hemorrhoids, or were included in this study. Subjective Mental Effort Questionnaire (SMEQ) and Local Experienced Discomfort (LED) scale were used to score the level of mental and physical stress among the operating surgeon, assistants, and the scrub nurse. Other parameters studied were the exposure of the operative site, patient comfort level, and position-related complications.

Results: Thirty patients were operated in each position. Mean \pm standard deviation of jackknife prone vs. lithotomy surgeon SMEQ score $(15.6 \pm 10.4$ vs. $107.0 \pm 11.5, \mathrm{P}<0.05)$ and LED score $(1.8 \pm 1.5$ vs. $6.7 \pm 0.5, \mathrm{P}<0.05)$ were found to be statistically significant. Prone vs. lithotomy assistant SMEQ score $(29.1 \pm 13.1$ vs. $100.6 \pm 8.7, \mathrm{P}<0.05)$ and LED score $(4.6 \pm 1.1$ vs. $7.4 \pm 0.8, \mathrm{P}<0.05)$ were also found to be statistically significant. SMEQ $(10.0 \pm 0.0$ vs. $20.6 \pm 2.5, \mathrm{P}<0.05)$ and LED scores $(1.1 \pm 0.3$ vs. $3.3 \pm 0.5, \mathrm{P}<0.05)$ of scrub nurses and LED scores $(2.5 \pm 0.5$ vs. $6.3 \pm 0.7, \mathrm{P}<0.05)$ of patients were also statistically significant. Exposure of the operative site was significantly better in the prone position (5.0 vs. 2.1, $\mathrm{P}<0.05$ ).

Conclusion: Significantly better SMEQ, LED, and exposure score suggests the superiority of jackknife prone position over the lithotomy in terms of significantly less mental and physical stress to the operating surgeon, assistant, and scrub nurse; better ergonomics, and excellent exposure.

\section{Keywords: Ergonomics; Prone position; Lithotomy; Perianal procedure}

\section{INTRODUCTION}

Lithotomy and jackknife prone are the 2 commonly used positions for perianal surgeries, the former being conventionally more used [1]. It provides good exposure to the posterior face of the rectum and the rectovaginal septum in females. However, this position is theoretically less comfortable for the surgeon and the assistants. Besides, blood tends to pool to the operating site, and lighting is often challenging [2]. Studies have shown lesser esti-

Received: Sep 19, 2020 - Revised: Dec 15, 2020 - Accepted: Dec 16, 2020 Correspondence to: Pankaj Kumar, M.S.

Department of General Surgery, All India Institute of Medical Sciences

(AllMS) Bhubaneswar, Sijua, Patrapada, Bhubaneswar 751019, India

E-mail: drpkushwaha@gmail.com

ORCID: https://orcid.org/0000-0003-2449-5514

(C) 2022 The Korean Society of Coloproctology

This is an open-access article distributed under the terms of the Creative Commons Attribution NonCommercial License (https://creativecommons.org/licenses/by-nc/4.0) which permits unrestricted non-

commercial use, distribution, and reproduction in any medium, provided the original work is properly cited. mated blood loss and operative time while performing abdominoperineal resection (APR) in jackknife prone position [2-5]. The rates of perforation and positive circumferential resection margin, too, are found to be lower in this position [4]. Although researchers unanimously agree that ergonomics in lithotomy position is unsatisfactory for both the surgeon and the assistant, no attempt has been made to compare these positions concerning ergonomics. The literature is sparse regarding the advantages of these positions over one another. No guidelines exist to date regarding the preference of the patient's position based on the anatomical location of perianal pathology. In our experience, the lithotomy position is uncomfortable to the patients, surgeons, and the assistants, mainly due to the limited exposure, low lighting, and awkward position of the operating team and patient. Comorbidities like osteoarthritis, previous arthrodesis, joint stiffness, and previous spine surgery can prohibit the use of this position [6]. Another common problem encountered during such operations in the lithotomy position is the constant soiling of the operative field with blood during dissection and inadequate exposure to bulky gluteal 
muscles. The surgeon has to bend, and assistants have to twist and turn to handle the operative site properly. This poor ergonomics theoretically affects both the performance and physical health of the surgeon and the assistants. The nursing assistant usually stands behind the surgeon and passes the instruments over the shoulder of the surgeon. It can be a potential threat of instrumentrelated injuries.

In contrast, the jackknife prone position can potentially provide excellent exposure, better ergonomics, exchange of instruments under vision, and avoid pooling blood in the operative field. Earlier, we use to perform surgeries of benign anorectal diseases like fissure, fistula, and hemorrhoids in a lithotomy position, but gradually have shifted to the jackknife prone position. Dr. Louis Buie first popularized the jackknife prone position for colorectal procedures [1]. Patients lie in the jackknife prone position with iliac crest positioned below the break in the operating table. However, it is not necessary to break the table for most of the anorectal procedures. We also keep the legs of the patient split, and buttocks are held apart by elastic tape. The surgeon stands between the patient's legs, and the assistant and nursing staff on the patient's side. We planned this study to compare the jackknife prone position with lithotomy in terms of ergonomics, complications, and comfort levels of the surgeon, assistant, and patients.

\section{METHODS}

The Institute Ethical Committee approved this prospective study, and we registered the trial with the clinical trial registry of India (CTRI/2019/12/022347). Written consent was obtained from all participants.

The enrolment started in December 2019, and the last patient was recruited in March 2020. The patients aged 18 years or older who were scheduled to undergo perianal surgery for fistula in ano, hemorrhoids, and fissure in ano were evaluated for inclusion in the study. The patients with contraindications to either positions or declining consent or were not able to understand the nature of the study were excluded. In the absence of previous similar trials, we planned this pilot study.

We included 30 patients in each group. The sample size was calculated based on the study by Browne [7]. The randomization scheme was generated by using the website of http://www.randomization.com. The allocation sequence was concealed from the operating surgeon using a double, opaque sealed envelope, and disclosure was declared in the operation theatre just before the patient's positioning. Patients were operated in the jackknife prone or lithotomy position as per the randomization sequence. All the patients underwent the operation by a single surgeon experienced in perianal surgery (about 100 surgeries annually). The surgeon operated with one assistant on one side of the patient and a scrub nurse on the opposite side. A total of 11 residents (assistants) and 7 scrub nurses participated in the study. The operating surgeon explained the study and case record form just before the beginning of the procedure. They were instructed to complete the data collection forms immediately after the procedure. The operating surgeon ensured that the assistants and the scrub nurses could not mutually discuss their responses.

The patients operated in the lithotomy position were made to lie supine with buttocks at the table's lower break. The hip joints were flexed $90^{\circ}$ and abducted at $30^{\circ}$ and knees, bent $70^{\circ}$ to $90^{\circ}$. The calves and lower thighs were supported on a padded leg shell, and the hands tucked at the side. The buttocks were lifted from the table with padded supports. In contrast, while operating the patients in jackknife prone position, patients were made to lie in the jackknife prone position with iliac crest lying below the operating table's break. We also split the patients' legs by $40^{\circ}$ to $60^{\circ}$, and buttocks were held apart by elastic tape. Adequate paddings were provided at the pressure areas to avoid neuromuscular complications. The patients were allowed to keep their hands in a comfortable position away from the area of interest. However, due care was taken to secure the intravenous cannula and other attachments.

Subjective Mental Effort Questionnaire (SMEQ) and the Local Experienced Discomfort (LED) scale were used to score the level of mental and physical stress among the operating surgeon and the assistants (Figs. 1, 2). The SMEQ is a cognitive workload questionnaire with a scale of 0 to 150 points to identify the amount of effort invested during a procedure [8]. Nine scale markers with verbal statements ranging from "no effort at all" to "an exceptional amount of effort" are displayed in the SMEQ diagram. The choice of statements and their scale locations are empirically derived. The LED scale allows participants to express their physical dis-

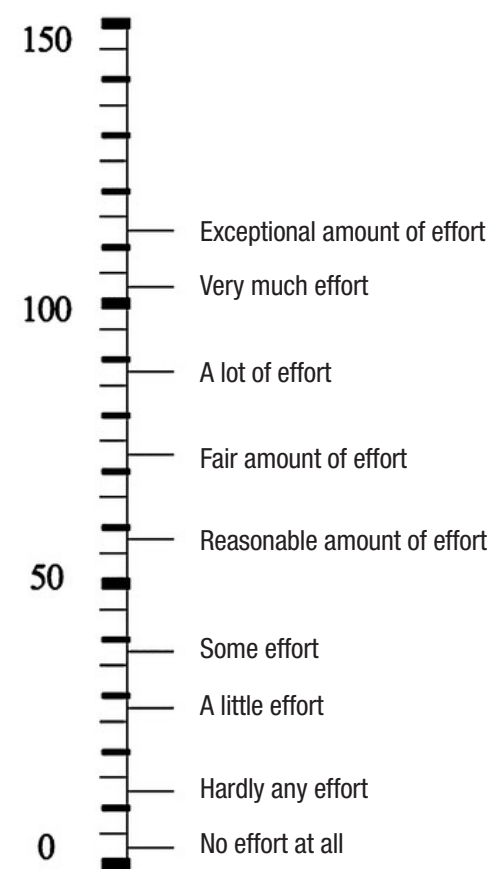

Fig. 1. Subjective Mental Effort Questionnaire scale. 

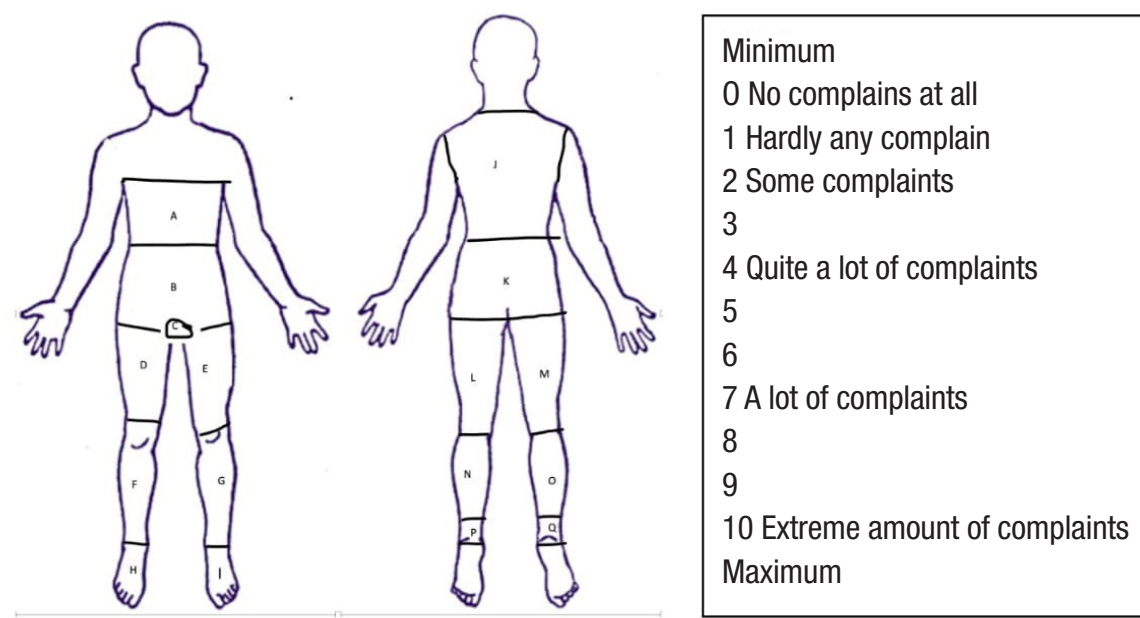

Fig. 2. Local Experienced Discomfort scale.

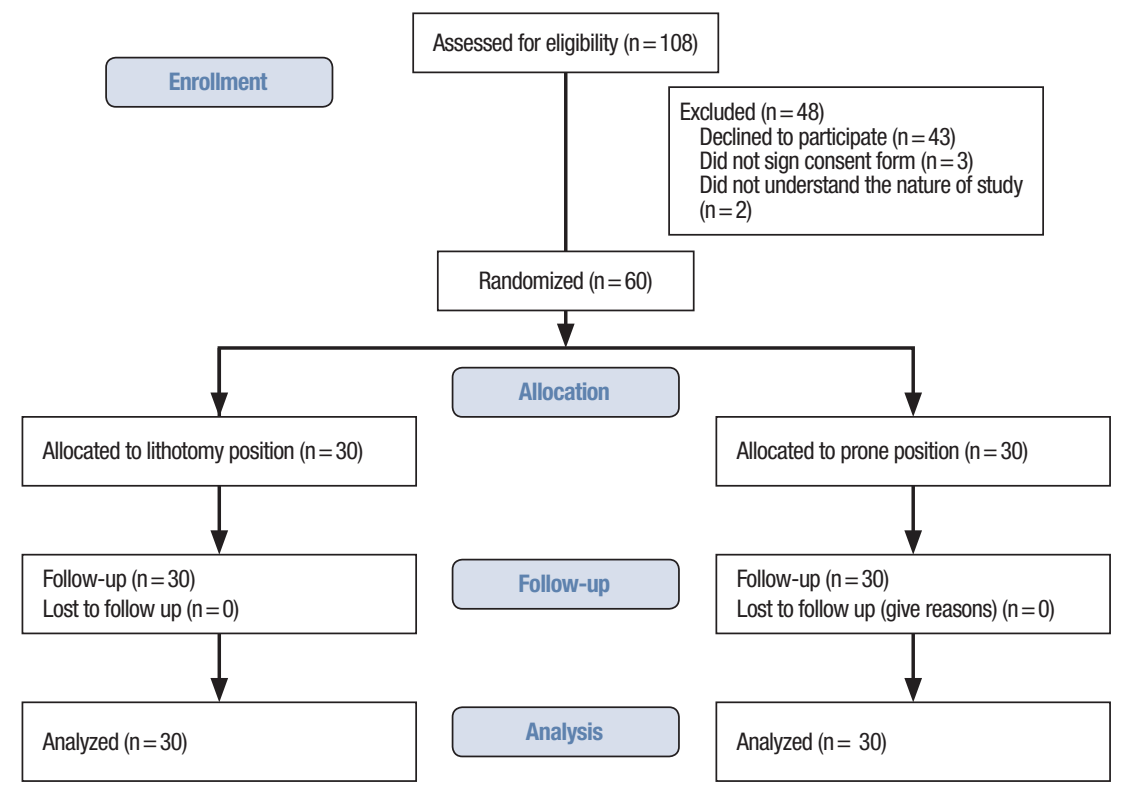

Fig. 3. CONSORT (Consolidated Standards of Reporting Trials) diagram for this study.

comfort during all the tasks [9]. On a scale of 0 to 10 points, the surgeon and the assistants rated their physical discomfort during the surgery. The duration of the operation, procedure, and position-related complications, and any need for a change in position was noted separately for different types of surgeries. The exposure of the operative site was measured on a scale of 1 to 5 (1, excellent; 2 , very good; 3 , good; 4 , average; 5 , poor) by the operating surgeon and the assistants. The comfort level of patients was also noted using the LED scale if operated under local anesthesia. The number of patients who could not be included in the study was also registered. Statistical analysis of both questionnaires was performed using a Mann-Whitney U-test. The t-tests analyzed the difference between the groups for continuous variables and chi- square tests for categorical variables. IBM SPSS Statistics ver. 25 (IBM Corp., Armonk, NY, USA) was used for analysis.

\section{RESULTS}

We evaluated a total of 108 patients for inclusion in the trial. Forty-three patients refused to participate; 3 refused to sign the written consent form, and 2 could not understand the nature of the study (Fig. 3). All 48 excluded patients did not have any contraindications for either position. Sixty patients were randomized equally for surgery in each of the positions. The baseline characteristics, including the disease frequency, were similar in either group (Table 1). The majority of patients operated on were male 
Table 1. Baseline characteristics

\begin{tabular}{|c|c|c|c|c|c|}
\hline \multirow{2}{*}{ Characteristic } & \multicolumn{3}{|c|}{ Applied method } & \multicolumn{2}{|c|}{ Procedure } \\
\hline & Jackknife prone & Lithotomy & P-value & Jackknife prone & Lithotomy \\
\hline Age (yr) & $42.0 \pm 10.2$ & $40.6 \pm 12.1$ & $0.629^{a}$ & & \\
\hline Body mass index $\left(\mathrm{kg} / \mathrm{m}^{2}\right)$ & $26.0 \pm 2.7$ & $27.0 \pm 3.2$ & $0.196^{a}$ & & \\
\hline Fistulas & 24 & 25 & $>0.99^{b}$ & & \\
\hline Supralevator & $1(4.2)$ & $0(0)$ & & LIFT-1 & NA \\
\hline Extra sphincteric & $0(0)$ & $0(0)$ & & NA & NA \\
\hline Hemorrhoids & 4 & 3 & $>0.99^{b}$ & & \\
\hline Grade 1 & $0(0)$ & $0(0)$ & & NA & NA \\
\hline Fissure & 1 & 2 & $0.618^{b}$ & LIS-1 & LIS-2 \\
\hline Anal stenosis & 1 & 0 & $>0.5^{b}$ & V-Y plasty-1 & NA \\
\hline
\end{tabular}

Values are presented as mean \pm standard deviation, number only, or number (\%).

FPR, fistulectomy with primary sphincter reconstruction; LIFT, ligation of intersphincteric fistula tract; ERAF, endorectal advancement flap; NA, not applicable; LIS, lateral internal sphincterotomy.

P-values were analyzed by ${ }^{a t-t e s t ~ a n d ~}{ }^{b}$ chi-square test.

Table 2. The scores of Subjective Mental Effort Questionnaire (SMEQ) and Local Experienced Discomfort (LED) scale

\begin{tabular}{lcc}
\hline Parameter & Jackknife prone & Lithotomy \\
\hline Surgeon & & \\
SMEQ & $15.6 \pm 10.4$ & $107.0 \pm 11.5$ \\
LED & $1.8 \pm 1.5$ & $6.7 \pm 0.5$ \\
Assistant & & \\
SMEQ & $29.1 \pm 13.1$ & $100.6 \pm 8.7$ \\
LED & $4.6 \pm 1.1$ & $7.4 \pm 0.8$ \\
Scrub nurse & & \\
SMEQ & $10.0 \pm 0.0$ & $20.6 \pm 2.5$ \\
LED & $1.1 \pm 0.3$ & $3.3 \pm 0.5$ \\
LED score of the patients & $2.5 \pm 0.5$ & $6.3 \pm 0.7$ \\
Exposure of the operative site & $5.0 \pm 0.0$ & $2.1 \pm 0.4$ \\
\hline
\end{tabular}

Values are presented as mean \pm standard deviation.

All $\mathrm{P}<0.05$ (by t-test).

(91.6\%), and the mean age was $41.35 \pm 11.09$ years. A significant difference in the SMEQ scores and the LED scores of the operating surgeon, assistants, and scrub nurses was observed between the groups (Table 2). We did not measure the SMEQ score of the patients. The LED score of the patient was also found to be significantly different in the 2 groups. The operative site's exposure was considerably better in the jackknife prone position (5.0 vs. 2.1, $\mathrm{P}<0.05$ ). The duration of the surgery operated under a jackknife prone position was 12 minutes shorter than in lithotomy position (mean \pm standard deviation: $33.93 \pm 7.38$ vs. $45.4 \pm 9.61$, respectively; $\mathrm{P}<0.05)$. There were no complications in either position.

\section{DISCUSSION}

Ergonomics deals with the study of human activities and behavior in the working environment [10]. It is one of the critical factors contributing to the outcome of any surgery. A lot has been discussed about the ergonomics in laparoscopy. However, ergonomics is equally vital in open operations to enhance the performance and achieve a better outcome [11]. Proper positioning of the patient is one of the crucial principles of ergonomics. Anorectal procedures are usually performed in 1 of the 3 positions: jackknife prone, lithotomy, and lateral. Out of these 3 positions, lithotomy is most popular, followed by a jackknife prone position. The popularity of lithotomy appears more to be a consequence of how we have been trained instead of better visibility or outcome. Nivatvongs et al. [12] have tried to suggest the type of anesthesia and the patient's position based on the shape of the buttock. The lithotomy position is recommended for type A buttock (the mounds of the buttock make a low and gentle slope with the anal verge) and type $C$ buttock (anus is located more anteriorly than usually). They suggested that a jackknife prone position is more 
suitable for type B buttocks. However, we randomized the patients without differentiating the patients based on the shape of the buttock. Our study, however, does not support this hypothesis. The jackknife prone position seems to be better than lithotomy irrespective of the shape of the buttock.

Patients have to be turned to the prone position after intubation while operating the patient under the jackknife prone position, during which there is always a chance of accidental dislodgement of the endotracheal tube. In this situation, patients have to be turned again to a supine position to secure the airway. Although these events are rare nowadays with expert anesthesia care, the airways need to be secured with extreme care. Apart from this demerit, there are several advantages of jackknife prone position over lithotomy. The surgeons operating in lithotomy position remained stuck between the legs of the patients, with restricted mobility. The assistants also have restricted access to the operation site. The assistants have to stand in an awkward position to keep the operative area well exposed, causing physical and mental discomfort. The soiling of the operative field with blood adds to the limitations of this position. In contrast, in a jackknife prone position, the surgeon and assistants stand comfortably by the patient's side, without restricting mobility.

Despite several advantages of jackknife prone position over the lithotomy, acceptance of jackknife prone position for perianal procedures is extremely low, at least in our center. Researchers have compared the operation time, blood loss, rectal perforation rate, circumferential resection margin, and oncological outcome of APR, in lithotomy and jackknife prone position, with several advantages in the later [2-5]. However, we could not find any study comparing these 2 positions regarding the ergonomics, comfort of the surgical team, and position-related complications. We routinely operate perianal procedures in a modified jackknife prone position. In this position, the patient is made to lie prone after induction of anesthesia. The legs are abducted, at the level of the hip joint, as far as possible. We try to achieve an angle of $60^{\circ}$ between the 2 limbs. A pillow/soft pad is put below the shoulder and hip to lift off the pressure on the abdomen. The compressive force over the stomach should be avoided to prevent cephalic displacement of the diaphragm, which may cause an increase in intrathoracic pressure and reduced residual pulmonary capacity. Physiological dysfunction is minimal and well-tolerated unless the patient has preexisting cardiopulmonary problems $[13,14]$. Both lithotomy and jackknife prone positions can produce neurological complications due to nerve compression; if the surgeries are prolonged and adequate padding is not done [13].

Most of our benign perianal procedures are performed under perianal block as a daycare procedure. We resort to regional or general anesthesia if the patients refuse to operate under local anesthesia or find the procedure unsuitable for completing under local anesthesia.

The operating surgeon's physical stress level, measured by the LED scale, was significantly better while operating the patients in a jackknife prone position. The operating surgeon operated on the patients in lithotomy by sitting on a stool between the patient's legs. However, he had to twist, extend, and bend multiple times to either approach the operating site or get the desired instruments. The LED scale reflects the physical discomfort. In contrast, the surgeon comfortably operated on the patient by standing between the patient's legs and lying in a jackknife prone position. Constant soiling of the field by blood and inadequate exposure of the operative field in the lithotomy position, as reflected by the exposure scale [1-5], led to mental discomfort. The SMEQ score used to measure the mental stress level showed a statistically significant difference favoring the jackknife prone position.

The observations were similar to both the assistants and the scrub nurses. The assistants either stand behind the surgeon or on one side of the limb when operated in lithotomy. When they are standing behind the surgeon, the vision is obstructed by the surgeon and by the lower limb when standing on one side of the patient. In both situations, it gets difficult for the assistant to assist actively. Their mental and physical discomfort increases further in the lithotomy position due to improper lighting and exposure.

Scrub nurses also reported significantly lower physical and mental stress levels in a jackknife prone position. In lithotomy, the scrub nurse stands behind the surgeon with the surgical tray and passes the instruments and mops over the shoulder of the surgeon, potentially compromising the sterility. It also increases the chances of needle stick injury and other injuries related to the instruments. The entire surgical team gets enough space to visualize the surgical field and assist actively in the jackknife prone position.

Interestingly, patients also reported significantly lower physical stress levels in jackknife prone positions. Over $70 \%$ of patients operated in lithotomy complained of mild pain and numbness over the calves. Eight patients (26.6\%), who got operated on in a jackknife prone position, complained of mild neck pain intraoperatively. None had any intraoperative or postoperative complications in either group. However, since all the patients were operated on under local anesthesia, this finding may not be extrapolated. A study with a more significant number of patients being operated on under anesthesia can help to answer this question.

The operating time was 12 minutes shorter in jackknife prone than in the lithotomy position. This decrease in the time is probably because the operative site's exposure was significantly better in a jackknife prone position; both the surgeon and the assistants were less stressed and participated actively in the procedure. There is also less soiling of the operative site with blood.

We faced difficulty in recruiting the patient for this trial. We explained that one position could be more uncomfortable than the other to them and the surgical team. It also revealed that the chances of complications in one position could be theoretically more than the other. The patients asked about the preference of the operating surgeon and the usual position adopted by him. Since our choice is the jackknife prone position, many patients opted for a jackknife prone position and refused randomization, 
which could be a serious limitation of this study. Secondly, a single surgeon operated on all the patients, and his inherent preference was to the jackknife prone position. Hence there is a fair chance of a bias toward the jackknife prone position. However, it's worth mentioning that the assistants and the nursing assistant were exposed equally to both situations. A total of 11 surgical residents and 7 nursing staff participated in the study, which adds to the merit of the study. Hence, even if the surgeon's scores are biased, the same may not be valid for the assistants.

Similarly, patient scores should also be free of any bias. Since the majority of the patients were young males, this could lead to selection bias. However, it is essential to realize that around $80 \%$ of the patients who attend our coloproctology clinic are young males. So, it is not possible to overcome this bias. We suggest a study with a large sample size to include many females and elderly patients to reduce the selection bias. More number of surgeons can also be included in this larger study to reduce preference-related bias.

The LED score developed by Corlett and Bishop [9] was modified for this study to include all possible pressure areas. We found it challenging to measure the stress level in the respective areas. Hence mean of the LED scores of the regions reported by the surgeon, assistants, and patients were considered for the analysis. This is another limitation of this study.

All the scores measured are subjective, and the ergonomics were not assessed objectively. Although this can be a limitation of the study, this does not devalue the study. This was a pilot study and can be repeated with the surgeons who do not prefer the jackknife prone position. A similar study can be planned with large sample size, and ergonomics/comfort can be measured objectively. van der Schatte Olivier et al. [15] have done a similar study to measure the comfort level objectively. Physiologic stress parameters were recorded using a wearable ambulatory monitoring system device. Average of 30 seconds recording of continuously monitored mean square of successive differences between consecutive heartbeats, preejection period (time of isovolumetric contraction), and average heart rate were compared between the 2 groups (laparoscopic vs. robotic surgery). However, the benefit of objectively assessing the comfort level using the cumbersome equipment seems to be of questionable value, considering the high cost of these tools. Our study's results suggested a jackknife prone position could be better than the lithotomy position in terms of intraoperative exposure and comfort level of the surgical team and the patients. But this statement cannot be extrapolated for all perianal procedures. Many procedures will still require the lithotomy position. The position should be decided based on the patient's condition, anatomical location of the disease, and the surgical procedure. For example, a jackknife prone position is not feasible for pregnant women and patients with ascites. It is also not suitable for a pathology near the scrotum. We prefer the jackknife prone position for hemorrhoids, fissures, fistulas, and anal stenosis.

To conclude, this pilot study results suggest that the jackknife prone position provides excellent exposure, better ergonomics, significantly less mental and physical stress to the operating surgeon, assistant, and scrub nurse compared to the lithotomy position. The jackknife prone position is also more comfortable for the patient being operated under local anesthesia. It can be preferred in all benign perianal procedures unless it is contraindicated. However, further studies are needed with a larger sample size to include the females and elderly patients, measure the ergonomics objectively by wearable devices, and include multiple surgeons to avoid biases.

\section{CONFLICT OF INTEREST}

No potential conflict of interest relevant to this article was reported.

\section{FUNDING}

None.

\section{REFERENCES}

1. Karulf RE, Goldberg SM, Bailey HR, Luchtefeld MA. Positioning the patient for anorectal surgical procedures. In: Bailey HR, Snyder MJ, editors. Ambulatory anorectal surgery [Internet]. New York: Springer; 2000 [cited 2020 Nov 5]. Available from: https:// doi.org/10.1007/978-1-4612-1196-9_4.

2. Liu P, Bao H, Zhang X, Zhang J, Ma L, Wang Y, et al. Better operative outcomes achieved with the prone jackknife vs. lithotomy position during abdominoperineal resection in patients with low rectal cancer. World J Surg Oncol 2015;13:39.

3. Mesquita-Neto JW, Mouzaihem H, Macedo FI, Heilbrun LK, Weaver DW, Kim S. Perioperative and oncological outcomes of abdominoperineal resection in the prone position vs the classic lithotomy position: a systematic review with meta-analysis. J Surg Oncol 2019;119:979-86.

4. McKechnie T, Lee Y, Springer JE, Doumouras AG, Hong D, Eskicioglu C. Prone compared with lithotomy for abdominoperineal resection: a systematic review and meta-analysis. J Surg Res 2019; 243:469-80

5. de Campos-Lobato LF, Stocchi L, Dietz DW, Lavery IC, Fazio VW, Kalady MF. Prone or lithotomy positioning during an abdominoperineal resection for rectal cancer results in comparable oncologic outcomes. Dis Colon Rectum 2011;54:939-46.

6. Fleisch MC, Bremerich D, Schulte-Mattler W, Tannen A, Teichmann AT, Bader W, et al. Guideline of DGGG (S1-Level, AWMF Registry No.015/077, February 2015). Geburtshilfe Frauenheilkd 2015;75:792-807.

7. Browne RH. On the use of a pilot sample for sample size determination. Stat Med 1995;14:1933-40.

8. Zijlstra FR. Efficiency in work behaviour: a design approach for modern tools [Internet]. Delft, NL: Delft University Press; c1993 
[cited 2020 Aug 13]. Available from: https://repository.tudelft.nl/islandora/object/uuid\%3Ad97a028b-c3dc-4930-b2ab-a7877993a17f.

9. Corlett EN, Bishop RP. A technique for assessing postural discomfort. Ergonomics 1976;19:175-82.

10. Catanzarite T, Tan-Kim J, Whitcomb EL, Menefee S. Ergonomics in surgery: a review. Female Pelvic Med Reconstr Surg 2018;24:112.

11. Schlussel AT, Maykel JA. Ergonomics and musculoskeletal health of the surgeon. Clin Colon Rectal Surg 2019;32:424-34.

12. Nivatvongs S, Fang DT, Kennedy HL. The shape of the buttocks: a useful guide for selection of anesthesia and patient position in anorectal surgery. Dis Colon Rectum 1983;26:85-6.

13. Roig-Vila JV, García-Armengol J, Bruna-Esteban M, Redondo-
Cano C, Tornero-Ibáñez F, García-Aguado R. Operating position in colorectal surgery: the importance of the basics. Cir Esp 2009; 86:204-12.

14. Borodiciene J, Gudaityte J, Macas A. Lithotomy versus jack-knife position on haemodynamic parameters assessed by impedance cardiography during anorectal surgery under low dose spinal anaesthesia: a randomized controlled trial. BMC Anesthesiol 2015; 15:74.

15. van der Schatte Olivier RH, Van't Hullenaar CD, Ruurda JP, Broeders IA. Ergonomics, user comfort, and performance in standard and robot-assisted laparoscopic surgery. Surg Endosc 2009; 23:1365-71. 